\title{
Application of RBF neural networks for real-time pressure prediction in a Diesel engine
}

\author{
Katarzyna Bizon ${ }^{1}$, Gaetano Continillo $^{2 *}$, Ezio Mancaruso ${ }^{3}$, Bianca Maria Vaglieco ${ }^{3}$ \\ ${ }^{1}$ Institute of Chemical and Process Engineering, Cracow University of Technology, ul. Warszawska 24, 31-155 Kraków, Poland \\ ${ }^{2}$ Department of Engineering, Università degli Studi del Sannio, Piazza Roma 21, 81200 Benevento, Italy \\ ${ }^{3}$ Istituto Motori CNR, Via Marconi 8, 80125 Naples, Italy \\ *Corresponding author.E-mail: continillo@unisannio.it
}

Copyright $(92015$ Katarzyna Bizon et al. This is an open access article distributed under the Creative Commons Attribution License, which permits unrestricted use, distribution, and reproduction in any medium, provided the original work is properly cited.

\begin{abstract}
This study aims at building efficient and robust artificial neural networks (ANN) in order to reconstruct the in-cylinder pressure of a Diesel engine starting from the signal of a low-cost accelerometer placed on the engine block. The accelerometer is a perfect non-intrusive replacement for expensive probes and is prospectively suitable for production vehicles. In this view, the ANN is meant to be efficient in terms of response time. In addition, robustness is sought in order to provide flexibility in terms of operation parameters.

The neural network considered here is based on radial basis functions (RBF). The network is trained using measurements from a single cylinder Diesel engine operating under varying conditions. Training data are composed of time series from the accelerometer and corresponding measured in-cylinder pressure signals. The network parameters, including the spread parameter of the radial basis functions and the number of neurons, are used to optimize the network quality. The RBF network is then validated.

The results show good correspondence between the measured and the simulated pressure signal. The accuracy of the simulated pressure signals is analyzed in terms of mean square error and in terms of a number of parameters, such as pressure peak and mass burned fraction (MBF), and their angular locations. Robustness is sought with respect to changes in the engine parameters and in the nature of the fuel. The encouraging results indicate that the reconstruction model based on RBF neural networks can be incorporated in the design of fuel-independent real-time control of Diesel engines.
\end{abstract}

Keywords: Accelerometer; In-Cylinder Pressure; Internal Combustion Engine; Neural Networks; Radial Basis Functions.

\section{Introduction}

The study and development of sensors for the diagnosis of the combustion quality in internal-combustion engines have recently been receiving significant interest due to the general intention of the replacement of intrusive and relatively expensive devices. One of the most important parameters for engine diagnosis and control is the in-cylinder pressure [1]. Its measurement, in fact, requires the use of a pressure transducer [2], which is expensive and intrusive. On the other hand, a better solution would certainly be the use of accelerometer systems placed externally on the engine block, as long as they can indirectly provide information about the quality of the combustion process. Such a solution would be less expensive than in-cylinder pressure probes, since it neither requires any special mounting process, nor does it expose the probe to severe in-cylinder conditions. Particularly, a strong relation was observed between the combustion pressure signal, and engine block vibrations. It is thus logical to try to correlate combustion characteristics with the vibration signal monitored by accelerometers. Quantification of this relationship has been attempted earlier for Diesel engines [3-5], confirming that the information extracted from the accelerometer signal can be profitably used in the closed-loop control system of internal-combustion engines. The transformation of the vibration signal into in-cylinder pressure is not straightforward, in spite of the strong correlation. This is not only due to the strongly nonlinear character 
of this relation but also, apparently, to the fact that the vibration signal contains some noise introduced, among the others, by piston slaps and valve impacts. Given the high level of complication of the engine as a physical system, it seems impossible to determine a transfer function basing on conventional physical model analysis. Rather, empirical tools must be constructed. In this view, artificial neural networks (ANN), which mimic the learning process of biological neural systems, are recognized as a tool having a great flexibility in the approximation of non-linear mappings, and are characterized by a relatively simple structure of nodes, adjustable during the training stage. ANNs are capable to learn both the associations and patterns in the measured data, even in presence of noise and uncertainty. Especially the last feature is of great importance when one deals with data characterized by a relatively low signal-tonoise ratio, as it is in this case. Earlier studies have shown the potential of neural networks in internal-combustion engine diagnostics. Different types of neural network models have been used to model the relationship between the engine crankshaft speed and parameters derived from in-cylinder pressure [6,7]. The crankshaft speed coupled with the Fourier transforms of the vibration signal was also used in [8] as an input for network simulation of in-cylinder pressure. Some other applications to neural networks to engine systems include: the implementation of neural network models using the engine speed signal for fault diagnosis [9]; the simulation of the air-fuel ratio dynamics using recurrent neural networks [10]; the prediction of nitrogen oxide's concentration in the exhaust from in-cylinder pressure signals in Diesel engine based on a radial basis function (RBF) network [11]; an adaptive feed-forward control system of the start of combustion (SOC) using the in-cylinder pressure measurement in a Diesel engine as a feedback variable [12]. Beyond the neural approach, a number of different signal processing tools were implemented for the reconstruction of the pressure signal and other important engine parameters. A time-domain smoothing technique based on Laplace transform for the reconstruction of in-cylinder pressure from the vibration response measured externally on a single-cylinder diesel engine was proposed in [13]. Some other examples include the use of spectral analysis for incylinder pressure reconstruction based on instantaneous engine speed signal [14] or deconvolution methods, such as optimal inverse filtering of cyclostationary processes, applied, for instance, in [15] for the reconstruction of the incylinder pressure from vibration measurements.

The present study aims at building an efficient and robust ANN method to determine the in-cylinder pressure signal starting from the vibration signal. Starting from the approach of [8], robustness is sought and verified with respect to changes in the engine parameters and in the nature of the fuel. The resulting neural network is then coded and incorporated in a commercial indicating system, to demonstrate its real-time capabilities. Basing on the present study, it would then be possible to design fuel-independent real-time control systems.

\section{Experimental equipment and procedure}

In order to study the potential of the RBF neural networks in the prediction of Diesel engine in-cylinder pressure from the engine block vibration signal, a number of tests were conducted on a single-cylinder (SC) research Diesel engine.

Table 1: Engine and Injection System Specifications

\begin{tabular}{ll}
\hline Engine type & 4-stroke single-cylinder \\
\hline Bore & $8.5 \mathrm{~cm}$ \\
Stroke & $9.2 \mathrm{~cm}$ \\
Displacement & $522 \mathrm{~cm}^{3}$ \\
Compression ratio & $16.5: 1$ \\
Injection system & Common Rail \\
Injector type & Solenoid driven \\
Number of holes & 7 \\
Cone angle of fuel jet axis & $148^{\circ}$ \\
Hole diameter & $0.141 \mathrm{~mm}$ \\
Rated flow @ 100 bar & $440 \mathrm{~cm}^{3} / 30 \mathrm{~s}$ \\
\hline
\end{tabular}

The single-cylinder research engine is equipped with the combustion system architecture and the Common Rail (CR) injection system of a 2 liter four-cylinder production engine (details in Tab. 1). The CR system is managed by an open electronic control unit (ECU) which allows controlling the number of injections per cycle, the injection pressure, the start and duration of injection, and the dwell time between consecutive injections. The vibrations of the engine were monitored by means of a low-cost, low power linear capacitive accelerometer, manufactured by STMicroelectronics ${ }^{\circledR}$. The accelerometer employed has a full scale of $\pm 2.0 \mathrm{~g}$ and is capable of measuring accelerations over a bandwidth of $1.5 \mathrm{kHz}$ for all axes, has a nominal sensitivity of $0.66 \mathrm{~V} / \mathrm{g}$, and is guaranteed to operate over a temperature range of $-40 /+85{ }^{\circ} \mathrm{C}$. The accelerometer was glued onto the upper surface of the cylinder, near the cylinder head and under the exhaust manifold, as shown in Figure 1. This location of the accelerometer on the engine head was chosen - from a number of different tested locations - to provide good accessibility and low sensitivity to other vibration sources. To acquire the in-cylinder pressure, a piezoelectric pressure transducer was placed in the glow plug seat of the engine head, namely an AVL GH13P with measuring range 0-250 bar and nominal sensitivity of 16 PC/ bar. A Hall-effect sensor was applied to the line of the solenoid current, to monitor the injection signal. 


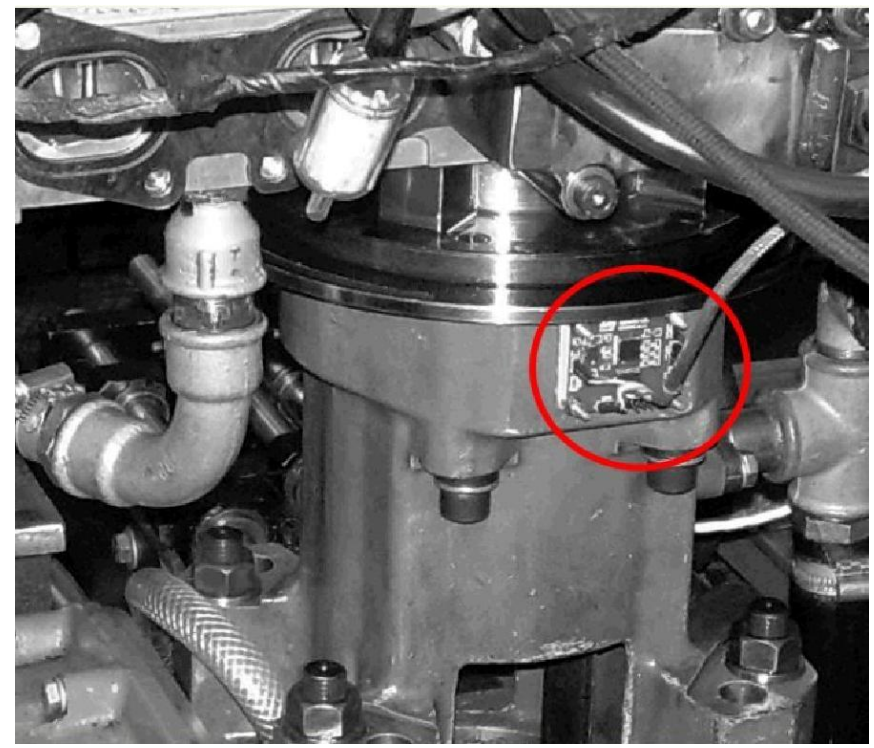

Fig. 1: Photograph of Experimental Setup Showing the Location of Accelerometer.

Aim of the work is to build a robust ANN, being able to predict the in-cylinder pressure from the acceleration signal for varying operating conditions, and thus guarantee flexibility in terms of operation parameters. In this view, measurements were made at different engine operating points (OP) which differ from each other in one or more of the following conditions: engine speed, type of fuel and injection strategy. Particularly, the engine (Tab. 2) was run in continuous mode at several engine speeds and with two different fuels: commercial Diesel fuel (OP1, OP2, OP3, and OP5) and rapeseed methyl ester (RME) (OP4, OP6). For the first operating condition, OP1, a typical HCCI (homogeneous charge compression ignition) strategy was chosen, employing five early injections. The other selected engine operating conditions were extracted from the ECU map of a Euro 5 passenger car engine, representing the conditions at $1000 \mathrm{rpm}$ idle (OP2), $1500 \mathrm{rpm}$ at 2 bars of BMEP (brake mean effective pressure) (OP3 and OP4), and $2000 \mathrm{rpm}$ at 5 bars of BMEP (OP5 and OP6). For OP2-OP6, the implemented injection strategies consisted of Pilot+Main pulses and were managed via the ECU. For each operating point, the in-cylinder pressure, the injector drive current and the acceleration signal were acquired and recorded at $0.5^{\circ}$ crank angle (CA) increments for a large number (total 2238) of fired engine cycles.

Table 2: Engine Operating Conditions

\begin{tabular}{lllll}
\hline OP & Speed $[\mathrm{rpm}]$ & Pinj $[\mathrm{bar}]$ & Fuel & Injection strategy \\
\hline 1 & 1000 & 700 & Diesel & Pre+Pilot+Main+Post+After \\
2 & 1000 & 420 & Diesel & Pilot+Main \\
3 & 1500 & 615 & Diesel & Pilot+Main \\
4 & 1500 & 615 & RME & Pilot + Main \\
5 & 2000 & 900 & Diesel & Pilot + Main \\
6 & 2000 & 900 & RME & Pilot + Main \\
\hline
\end{tabular}

\section{Introduction}

A radial basis function (RBF) network is a variant of neural network [16] which, in its simplest configuration, is characterized by a feed-forward architecture consisting of an input layer and a single hidden layer interconnected to an output layer being a weighted sum of the hidden unit outputs (Fig. 2). More precisely, given an input vectorx, consisting of $\mathrm{m}$ input signals, and a set of weights $\omega_{0}(i=1, \ldots, N)$, with $\omega_{0}$ a bias term, the network output $y$ is given by:

$y=\sum_{i=1}^{N} \omega_{i} R_{i}(x)+\omega_{0}$

where $\mathrm{N}$ denotes the number of neurons in the hidden layer and $\mathrm{R}_{\mathrm{i}}$ are the activation functions formulated as:

$\mathrm{R}_{\mathrm{i}}(\mathrm{x})=\phi\left(\left\|\mathrm{x}-\mathrm{c}_{\mathrm{i}}\right\|\right)$ 


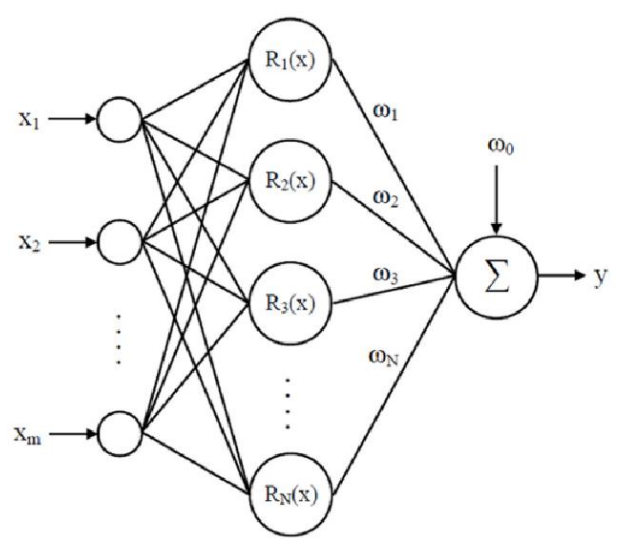

Fig. 2: Typical Architecture of an Artificial Neural Network.

where $\phi$ is the radial basis function providing the nonlinear feature of the model, and $c_{i}$ are so called RBF centers. Various functions have been tested as activation functions, including Gauss function, multiquadratic, inverse multiquadratic or thin plate spline functions $[17,18]$. In this work, the most popular RBF is adopted, i.e. the Gauss function, given by:

$\phi(r)=\exp \left(\frac{-r^{2}}{\sigma^{2}}\right)$

The variable $\mathrm{r}$ in Eq. 3 indicates the Euclidian distance between the input vector $\mathrm{x}$ and the center $\mathrm{c}_{\mathrm{i}}$, namely:

$r=\left\|x-c_{i}\right\|$

whereas $\sigma$ denotes a spread parameter, also called basis width parameter. The training of the network consists then in determining the centers of the activation functions $\phi$, the number $\mathrm{N}$ of the neurons in the network, the connection weights $\omega_{i}$, and the value of the spread parameter $\sigma$. To this aim, an optimization problem is solved that minimizes the distance between the available sample of the targets and the corresponding outputs of the network.

A number of methods exist for the selection of RBF centers $c_{i}$, with either a uniform or a random distribution of the centers across the input space being the simplest policies. Some more sophisticated methods can be used for a smarter selection of the centers, such as for example the $k$-means clustering method. The basic idea of this method is to distribute the centers according to the natural measure of the attractor, i.e. where the density of the data points is high, so the density of the centers will be [19]. In this work a recursive selection procedure of the centers is adopted, which consists of adding one center (neuron) at a time, basing on the error between the target and the output of the network. Namely, at the end of each training step, a new center is taken, given by the input vector characterized by the largest target-output distance. More neurons are added to the network until the mean squared error falls beneath an error goal or until a prescribed maximum number of neurons have been reached. When it comes to the choice of the spread value $\sigma$, which is crucial for the network performance [17], it can be observed that the larger the spread, the smoother the approximation will be, due to the properties of the Gauss function. This means that too large a spread will cause the need for a large number of neurons in order to fit a fast changing function. On the other hand, if the spread is too small, a large number of neurons will be required to fit a smooth function.

A desirable property for a model is generalization, i.e. the ability of the network of processing data that has never seen before. Generalization not only depends on the optimal choice of the network parameters (here number and values of centers $c_{i}$, and spread $\sigma$ ) but also on the choice of the most significant and informative pairs of input-target training samples. These can be identified by means of various active learning approaches [20]. Here a plain sampling strategy is used by picking events that are uniformly spaced in the set of the available data.

\section{Results and discussion}

\subsection{Experimental results}

The primary aim of this study is the reconstruction of the in-cylinder pressure of a Diesel engine. It is known that the accelerometer signal is affected by a number of superimposed mechanical events, including piston slap as well as opening and closure of intake and exhaust valves [5]. Hence, the accelerometer signal acquired under motored conditions can be assumed as a baseline. Then, in order to evaluate the influence of the combustion event on the variation of the engine block vibration, the accelerometer signal, with the relevant in-cylinder pressure during operation of the single-cylinder engine under motored conditions, is first acquired and reported in Figure $3 \mathrm{a}$. When moving to 
fired conditions, the vibration signal changes as shown in Figure 3b. Particularly, a substantial change with respect to the baseline signal can be observed in the vicinity of the peak pressure, due to the increase of engine vibrations resulting from the fast change of the in-cylinder pressure when combustion occurs.
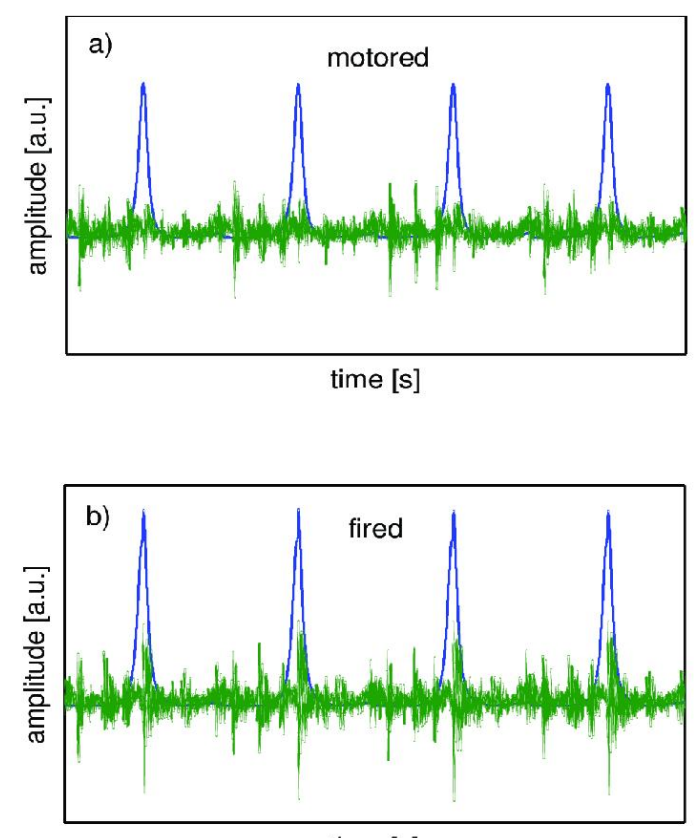

time [s]

Fig. 3: In-Cylinder Pressure and Accelerometer Signals in Motored (A) and Fired (B) at Engine Speed of 1500 Rpm with Commercial Diesel Fuel.
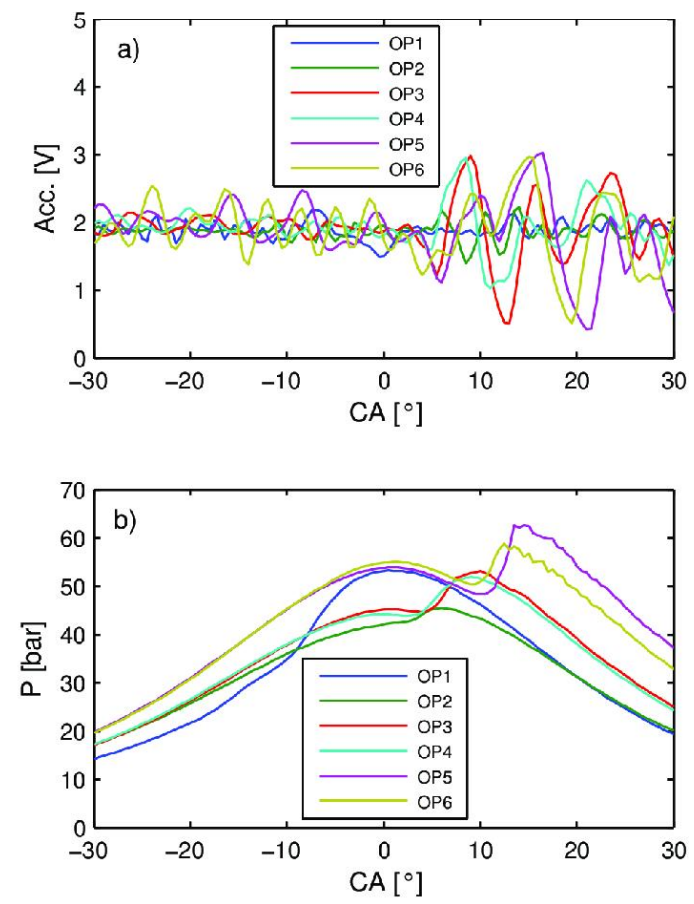

Fig. 4: Typical Accelerometer Signal (A) and in-Cylinder Pressure (B) for Different Engine Conditions (OP1-OP6).

Figure 4 reports some representative accelerometer (a) and corresponding in-cylinder pressure (b) signals for all considered operating points (OP1-OP6). At a first glance, it is relatively difficult to couple the corresponding pairs of signals. All signals are clipped to $\pm 30^{\circ} \mathrm{CA}$ (crank angle) around the top dead center (TDC) where all relevant events take place and the correlation between signals is the strongest. The clipped signals will be used successively in the determination of the optimal RBF model; the computational grounds of such choice will be clarified later on. It can be observed that OP1 and OP2, both at $1000 \mathrm{rpm}$, produce very different pressure profiles (Fig. 4b). This is due to the fact that OP1 is HCCI (an advanced combustion strategy) whereas OP2 corresponds to idle conditions of a conventional injection strategy. OP2 and OP3 $(1500 \mathrm{rpm})$ pressure profiles differ both in shape and in peak values, since higher engine speed and load were adopted with OP3. OP4 and OP6 only differ from OP3 and OP5 for the nature of the fuel 
used, i.e. commercial Diesel fuel and rapeseed methyl ester biofuel, respectively. This results into slightly different IMEP (indicated mean effective pressure) and pressure signals, more visible at the highest (2000 rpm) engine speeds. As already recalled, vibration signals manifest relatively high amplitude around the events of ignition and combustion (Fig. 4a), and appear to be strongly affected by the engine load, i.e. vibration signal peaks are higher as the engine load is increased. This confirms the very strong correlation between the combustion pressure signal, and the engine block vibrations, which, however, cannot be characterized in the straightforward manner due to evidently non-linear relationships: hence, the need for more sophisticated tools, such as artificial neural networks, emerges.

\subsection{RBF network training and validation for a single operating point}

The training of the neural model is first performed by using a single data set of measurements collected during the experiments. This allows for a detailed investigation of the influence of individual network parameters, i.e. spread of the RBF and number of centers, on the network performance, both during the training and the validation phase. Particularly, measurements are taken at operating point number 3 (OP3), corresponding to an engine speed of $1500 \mathrm{rpm}$ with commercial diesel fuel. The injection pressure was 615 bar with two injections per engine cycle, i.e. Pilot+Main. This experimental data set consists of 357 pairs of accelerometer (input of the network) and in-cylinder pressure signals (target of the network) acquired at consecutive fired cycles. The set was divided into two subsets: a training set of 179 cycles, and a validation set, made of the remaining 178 cycles. This initial test provides the estimate of the number of RBF centers (neurons) required to build a reliable model; subsequently, the influence of the spread parameter onto the network performance is investigated.

The most commonly used measure of network performance is the mean squared error (MSE) between the simulated and the actual signal. Figure 5a reports training performance of the neural network expressed in terms of MSE as a function of the number of neurons (RBF centers) in the network, for three arbitrarily chosen values of the spread parameter $\sigma$.
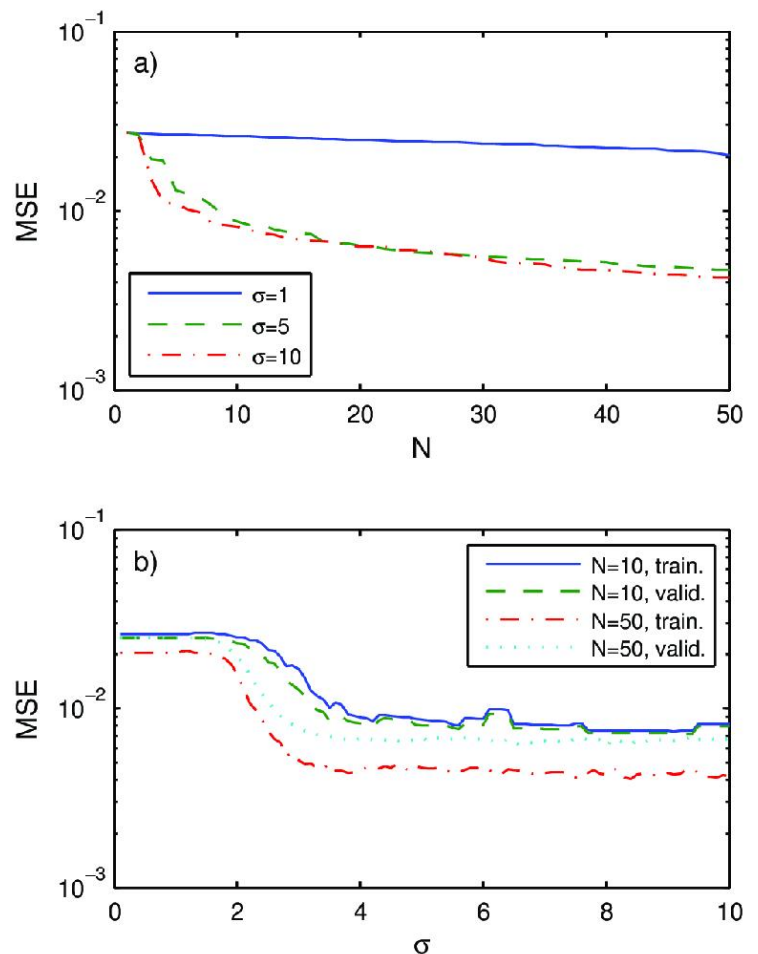

Fig. 5: Training Performance as a Function of the Number of Neurons N, at Various Values of the Spread Parameter $\sigma$ (A); Training and Validation Performance as a Function of the Spread Parameter $\sigma$ at Various Values of the Number of Neurons N (B).

It can be observed that an increase of the value of $\sigma$ leads to significant improvements in network accuracy, even with a very low number of neurons. In fact, both in the case of $\sigma=5$ and $\sigma=10$, a very fast convergence rate of MSE can be observed, achieving values lower than $10^{-2}$ bar $^{2}$ for networks employing as few as 10 neurons.

The low convergence rate of the error for a higher number of neurons $(\mathrm{N}>5)$ does not justify the adoption of too high a number of RFB centers in the network. The number of neurons should be retained as low as possible, both in view of the computational savings assured by the network employing fewer neurons, and in view of the potential risk of network overlearning - and of the consequently bad generalization performance, which usually characterizes systems with too many neurons. Figure $5 \mathrm{~b}$ shows the comparison of the training and validation MSE for $\mathrm{N}=10$ and $\mathrm{N}=50$, with spread parameter $\sigma \in[0.1,10]$ varying with increments of 0.1 . It can be observed that the network having 50 neurons manifests a distinct deterioration of performance when simulated on the validation set. For the case of 10 
neurons, on the contrary, there is no significant alteration between the training and the validation error. This makes it evident that selecting the number of centers and the spread value basing on the training set only does not guarantee an optimal model: therefore, both training and validation MSE are used as a selection criterion.

It was earlier observed that the network performance is not satisfactory for too low values of the spread. This is due to the character of the simulated signal being relatively smooth; hence the choice of too small a spread parameter would require too large a number of neurons to fit the signal. The MSE appears to remain of the same magnitude, irrespective of the number of neurons, for values of the basis spread $\sigma>4$ approximately. In a compromise between training and validation error, for the network employing $10 \mathrm{RBF}, \sigma=9.4$ can be chosen as the optimal value.

Figure 6 compares measured and simulated in-cylinder pressure signals for a cycle, arbitrarily chosen from the validation set. Excellent correspondence between the two signals is observed over the whole crank angle domain; nevertheless, the main practical interest lies in the range of $\pm 30^{\circ} \mathrm{CA}$ around $\mathrm{TDC}$, where all relevant events (i.e. pressure variation due to ignition and combustion) take place.

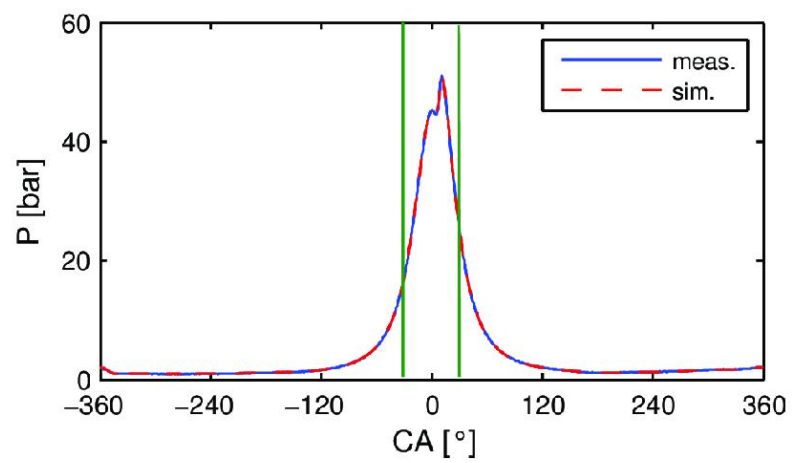

Fig. 6: A Typical Measured and Simulated in-Cylinder Pressure Cycle: RBF Network with 10 Neurons and Spread Value $\sigma=9.4$.

Thus it is now possible to focus on the analysis of network performance within the window marked by vertical lines in Fig. 6. As seen earlier, for the network employing $\mathrm{N}=10$ neurons and trained using the entire signals, i.e. in the range of $\pm 360^{\circ} \mathrm{CA}$ around TDC, the validation error is in the order of $10^{-2} \mathrm{bar}^{2}$, for $\sigma>4$ (Fig. 7, net1, valid.). If the error calculation is now confined to the clipped interval of $\pm 30^{\circ} \mathrm{CA}$, the validation error increases by about one order of magnitude (Fig. 7, net1, clipp.). This is caused by the fact that, in the first case, the calculated error is averaged over the entire domain of crank angle, which includes zones where the combustion phenomenon is not present: hence, the error is relatively small due to the almost constant pressure. In the second case the mean is calculated over a clipped interval of CA, where significant pressure variations are observed, thus the simulated signal is potentially affected by larger error. In this view, it can be concluded that the acquired signals should be better clipped initially to $\pm 30^{\circ} \mathrm{CA}$ range and in such form utilized for the training of the network. This leads not only to better network performance in the particular range of interest, but also to significant computational savings. In fact, both the training and the validation MSE of the second network, this time trained using clipped signals, reported in Figure 7 and denoted by net2, train. and net2, valid., respectively, are lower than the MSE calculated over the clipped interval of crank angle but employing a network trained using the entire signals (net1, clipp.). In addition to improvement in network accuracy, such procedure gives very significant savings - in terms of occupied memory and computational time - both when applied to training and to simulation: when the input-target pairs in their clipped form are used, the training phase is more than 10 times shorter than with the original signal, i.e. the training time reduces from 2.42 to $0.19 \mathrm{~s}$. In both cases, calculations times are very short and this difference seems not to be important; however, one has to remember that, in this illustrative case, only 179 pairs of training signals are used; this difference certainly gains importance when passing to global, multi-condition cases using large sets of training data. Even more important is the reduction of the simulation time, especially when it comes to real-time, online application of the neural network: clipping of the signal permits a much faster evaluation of the output signal from the input signal delivered to the network. Indeed, for a single cycle the simulation time diminishes from $2.5 \cdot 10^{-4}$ to $0.78 \cdot 10^{-4} \mathrm{~s}$. The optimal number of RBF for the clipped signals is found to be $\mathrm{N}=10$ also in this case, thus the detailed analysis of this aspect will not be repeated here. The optimal spread value, selected again by making a compromise between training and validation error, is found to be lower than before, namely $\sigma=4.9$. Figure 8 shows a MSE in the reconstruction of the in-cylinder pressure for the first 100 engine cycles from the validation set, being always lower than 0.2 bar $^{2}$. Successively, two measured and simulated parameters are compared, which are important for engine operation, i.e. the value of the peak pressure (maximum pressure) and its angular location (Fig. 9). As it can be seen in Figure 9a, the deviation between measured and simulated peak pressure values always remains within \pm 0.5 bars. The error in the prediction of the angular location of the peak (Fig 9b) does not exceed $1^{\circ} \mathrm{CA}$ for all cycles. 


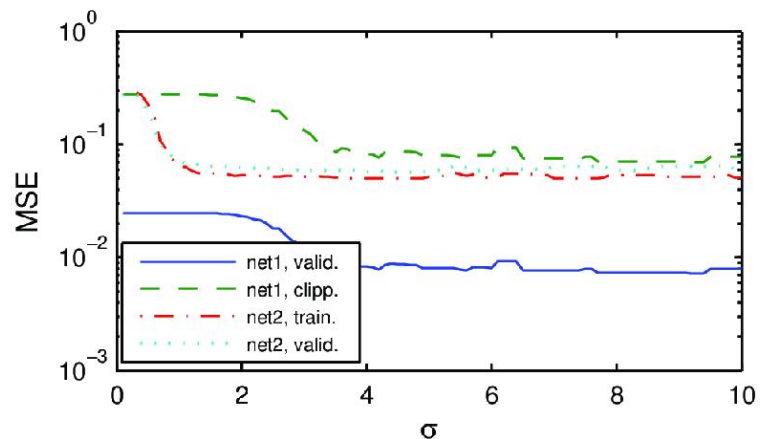

Fig. 7: Training and Validation Performance for Varying Spread Parameter $\sigma$.

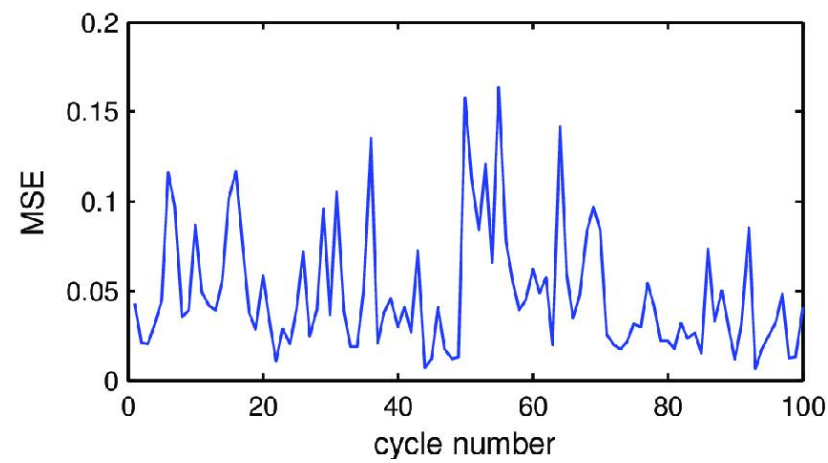

Fig. 8: Validation MSE for the Network Employing 10 Neurons.
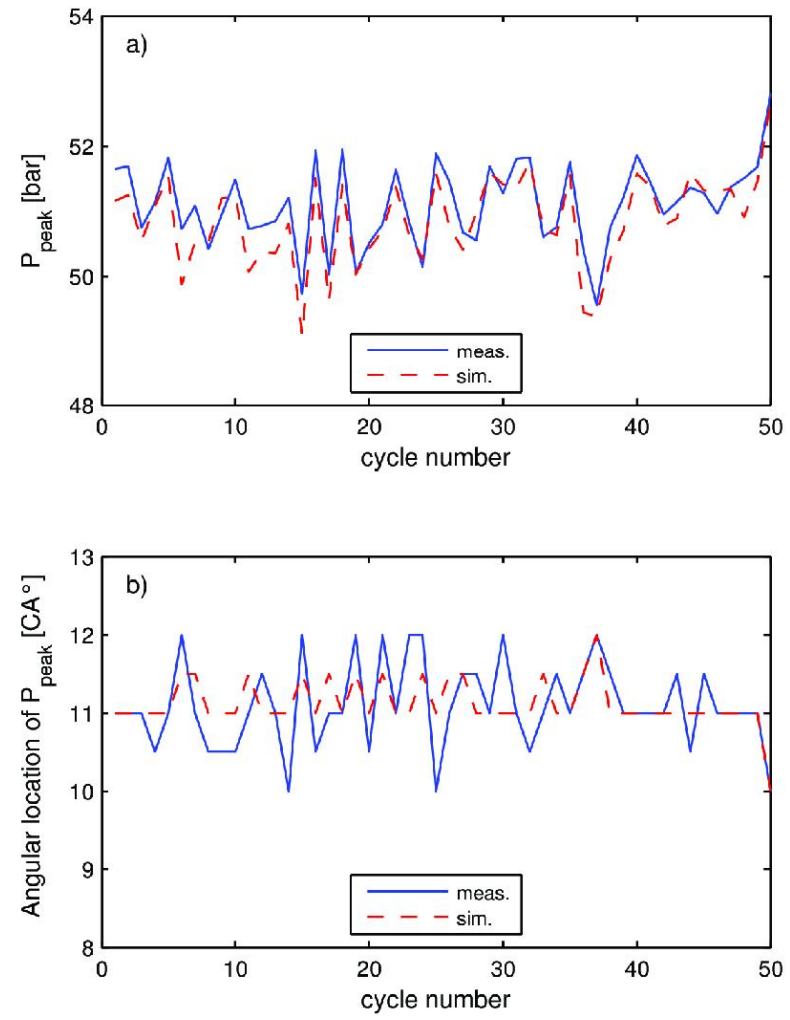

Fig. 9: Comparison between Measured and Simulated Peak Pressure Values (A) and between their Angular Locations (B).

\subsection{Robustness of the multi-condition RBF network}

A robust RBF neural network should provide accurate predictions of in-cylinder pressure for varying engine parameters and changes in the nature of the fuel. To this aim, the network is tuned by using a training set created from input-target pairs of accelerometer and pressure signals acquired at six different engine operating points (OP1-OP6; single-cylinder engine), described previously in Table 2. Exactly 100 cycles from each OP were selected, using a simple sampling 
strategy, namely picking events that are uniformly spaced in the set of the available data. As for the illustrative case, the optimal choice of the number of neurons was initially analyzed in terms of both training and validation MSE, in order to avoid the danger of overlearning that may result when using too many neurons. The best neural model proved to be the one employing 25 neurons.
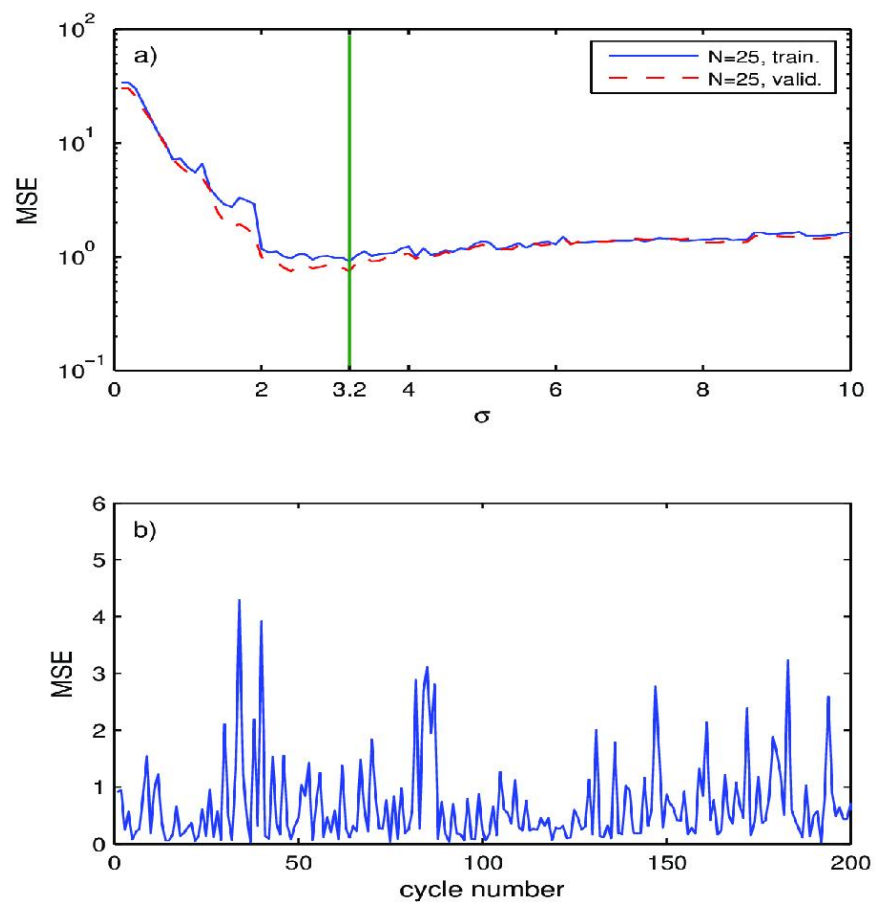

Fig. 10: Training and Validation Performance as a Function of the Spread Parameter $\sigma$ (A) and Validation MSE (B) for the Network Employing 25 Neurons.

Figure 10a illustrates the influence of the value of the spread, $\sigma$ on the training and validation MSE. As before, knowing that the best training MSE does not guarantee an optimal model in terms of generalization performance, the optimal value of the spread is sought by making a compromise between the training and the validation error, and selected as $\sigma=3.2$ (marked by the vertical line in Fig. 10a). For this value of the spread, the network is characterized by an overall training MSE equal to $0.918 \mathrm{bar}^{2}$ and by an overall validation MSE equal to $0.745 \mathrm{bar}^{2}$. This result confirms a very good generalization performance of the network.

Figure 10b shows individual values of the MSE, which vary in the range of $0.0183 \mathrm{bar}^{2}$ and $4.297 \mathrm{bar}^{2}$, for a number of arbitrarily chosen cycles from the validation set. It should be underlined that it is very important to accurately reconstruct not only the pressure peak value, but the whole pressure signal, commonly used in the determination of pressure derived parameters. For instance, the MBF50 (the time at which 50\% of the mass burned fraction is reached) and the SOC (Start Of Combustion) may be used in engine control.

\begin{tabular}{|c|c|c|c|c|}
\hline $\mathrm{OP}$ & $\begin{array}{l}\text { Pressure peak value } \\
\text { RMSE [bar] }\end{array}$ & Rel. err. [\%] & $\begin{array}{l}\text { Peak location } \\
\text { RMSE CA }\left[^{\circ}\right]\end{array}$ & $\begin{array}{l}\text { MBF50 } \\
\text { RMSE CA }\left[{ }^{\circ}\right]\end{array}$ \\
\hline 1 & 1.198 & 1.711 & 0.431 & 0.725 \\
\hline 2 & 0.803 & 1.353 & 0.499 & 3.448 \\
\hline 3 & 1.018 & 1.576 & 0.494 & 0.952 \\
\hline 4 & 0.925 & 1.380 & 0.604 & 0.786 \\
\hline 5 & 1.637 & 2.088 & 1.020 & 0.356 \\
\hline 6 & 1.355 & 1.784 & 0.548 & 0.784 \\
\hline
\end{tabular}

Figure 11 illustrates a comparison of measured and simulated in-cylinder pressure signals for a selection of cycles from the validation set, one for each operating point. There is very good agreement between simulated and measured signals, especially at lower engine speeds, such as 1000 and $1500 \mathrm{rpm}$ (Fig. 11a-d), over the entire combustion process. Slight discrepancies appear at $2000 \mathrm{rpm}$ (Fig. 11e-f), manifested by a much smoother character of the simulated with respect to the measured pressure. The performance of the network is summarized in Table 3 . The accuracy of the model is characterized in terms of RMSE and relative error in the peak pressure value prediction, and of RMSE in the prediction of angular location of pressure peak and MBF50, all calculated separately for the individual operating points. When pressure peak values are concerned, the highest values of the error (both RMSE and relative error) are observed again for the highest investigated engine speed of $2000 \mathrm{rpm}$, due to the smoothing introduced by the neural model. There is no 
big variation in the error on the pressure peak location among the various operating points: its value is - except for OP5 - as low as $\pm 0.5^{\circ} \mathrm{CA}$. In view of a possible use of the vibration signal in real time control of the Diesel engine, MBF50 is also calculated from the simulated pressure. MBF50 shows how the in-cylinder combustion progresses as a function of the crank angle. The MBF50 value is related to the thermal efficiency of the engine, to the peak cycle temperature and pressure, and to the exhaust emissions (Heywood 1988). Also in this case a satisfactory accuracy is achieved: namely, for 5 operating points the deviation in the simulation-delivered angular location of the MBF50 is of the order of $1^{\circ} \mathrm{CA}$ with respect to the value determined from the measured pressure. A significantly higher value of the deviation is noted for OP2 $\left(\sim 3.5^{\circ} \mathrm{CA}\right)$. This is due to the intrinsically unstable nature of the idle regime, for which - by the way there is little if any need of pressure-based real time engine control.
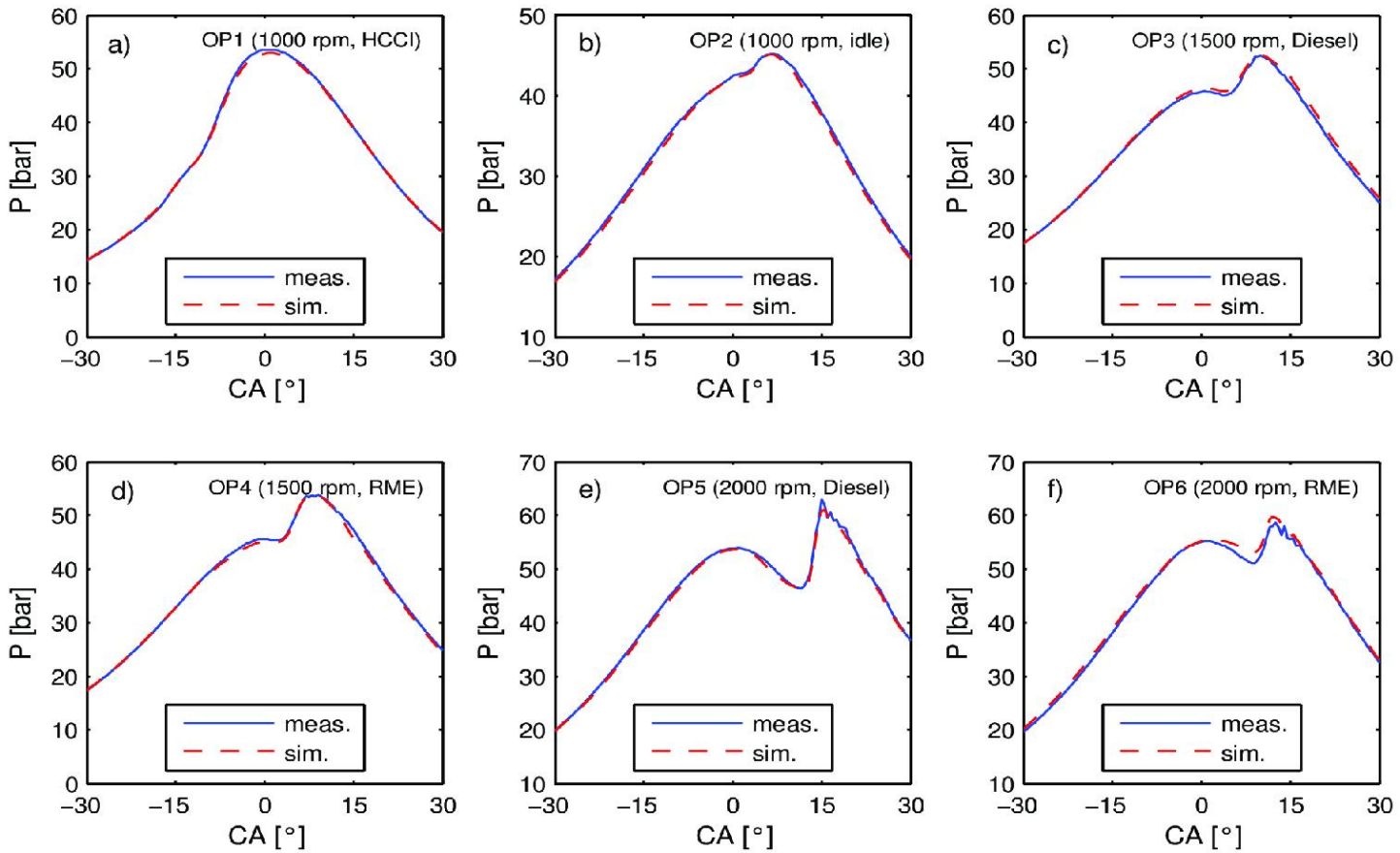

Fig. 11: Measured and Simulated in-Cylinder Pressure at Different Engine Conditions (OP1-OP6).

\subsection{Online pressure prediction}

Proper processing of the engine vibration signal by a neural model allows a very accurate estimation of some important combustion parameters, among which the pressure peak angular location and the MBF50. This is very promising for both diagnosis and control of the engine, without having to employ an in-cylinder pressure transducer [5]. With the aim of the application of a neural model for control purposes, the online application of the network for engine supervision was attempted. The model developed for the single-cylinder engine was implemented in the form of a macro in AVL IndiCom $^{\mathrm{TM}}$, a commonly used indicating system for internal-combustion engines. Using the acquired vibration signal as an input, the neural network macro permits real time simulation of the in-cylinder pressure and estimation of the mentioned pressure-derived parameters, i.e. pressure peak location and MFB50. Figure 12 reports screenshots of the supervision software demonstrating the functionality of the implemented macro for two engine speeds: $1500 \mathrm{rpm}$ (Fig. 12a) and $2000 \mathrm{rpm}$ (Fig. 12b). Red dashed lines denote measured in-cylinder pressure while blue solid lines denote the online reconstructed in-cylinder pressure by means of the neural network: in both cases, very good accuracy is demonstrated for the single-cycle reconstruction. The overall network performance for a number of cycles is illustrated in Figure 13 and in Table 4. Figure 13 shows values of MSE of in-cylinder pressure reconstruction calculated for 200 consecutive fired cycles for the engine operating at $1500 \mathrm{rpm}$ (Fig. 13a) and $2000 \mathrm{rpm}$ (Fig. 13b). The faster regime (2000 rpm) exhibits a progressive increase of the MSE with time, probably due to the increase in the thermal load of the combustion chamber.

Table 4: Neural Model Performance in the Online Application, for the Engine Operating at the Speed of 1500 Rpm and 2000 Rpm, Using Commercial Diesel Fuel.

\begin{tabular}{lllll}
\hline \multirow{2}{*}{ Engine speed $[\mathrm{rpm}]$} & \multicolumn{2}{l}{ Pressure peak value } & Peak location & MBF50 \\
& RMSE [bar] & Rel. err. [\%] & RMSE CA [ $\left.{ }^{\circ}\right]$ & RMSE CA [ $^{\circ}$ ] \\
\hline 1500 & 1.954 & 3.495 & 0.606 & 0.348 \\
2000 & 1.066 & 1.426 & 0.682 & 0.268 \\
\hline
\end{tabular}



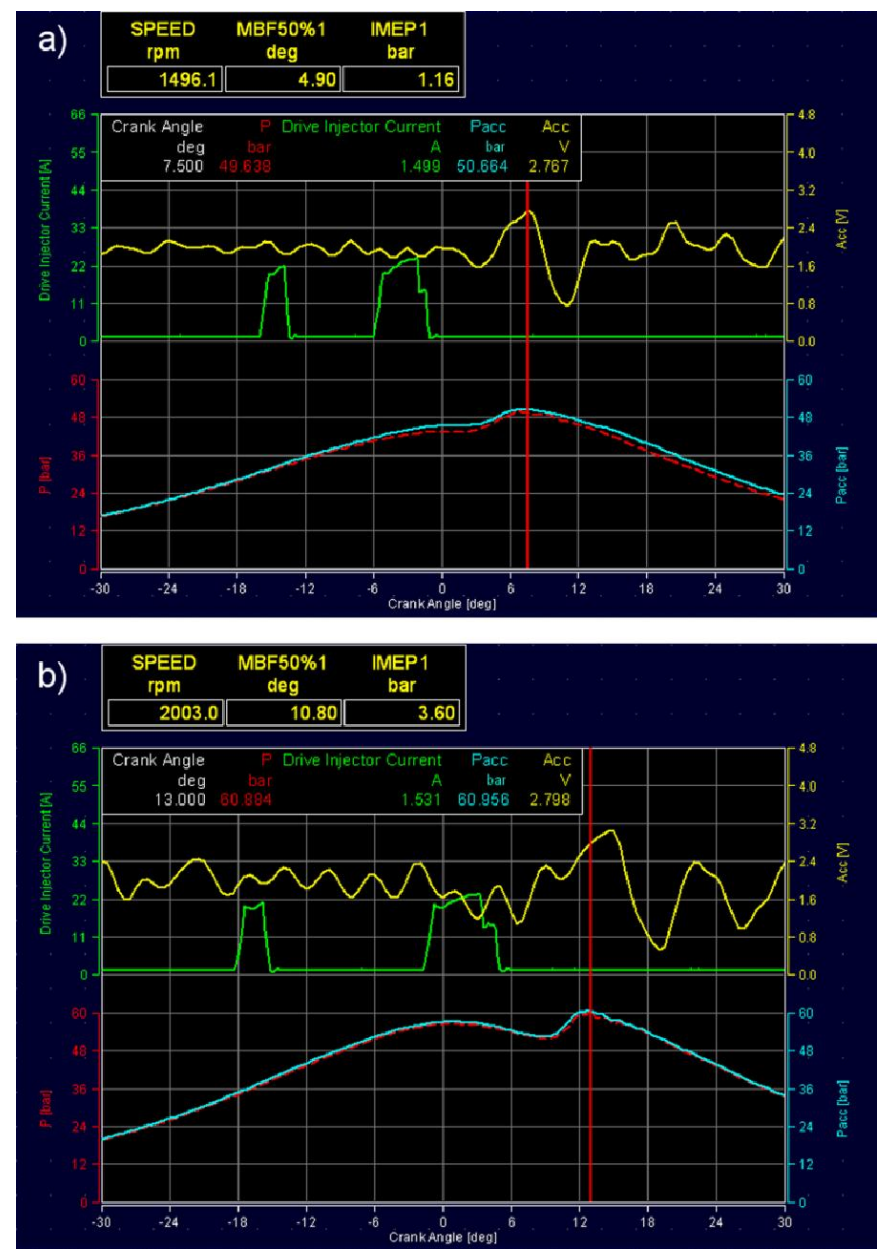

Fig. 12: Online Measured (in-Cylinder Pressure, Injector Drive Current and Accelerometer) and Simulated (in-Cylinder Pressure) Signals for SingleCylinder Engine Operating at 1500 Rpm (A) and 2000 Rpm (B).
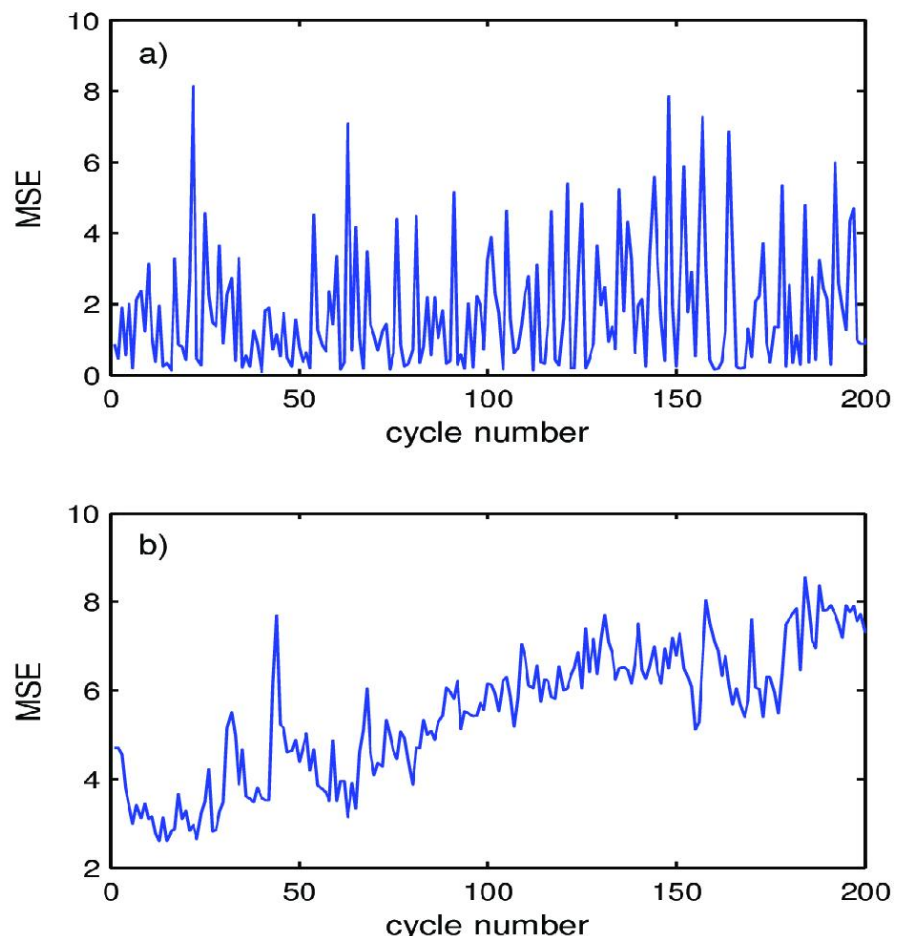

Fig. 13: MSE for Online in-Cylinder Pressure Reconstruction for the Network Employing 25 Neurons and Engine Operating at 1500 Rpm (A) and $2000 \mathrm{Rpm}(\mathrm{B})$. 


\section{Conclusions}

A radial basis function artificial neural network was developed for the prediction of the in-cylinder pressure as well as some pressure-derived engine parameters, namely angular locations of peak pressure and MBF50. The network uses as an input the vibration signal from an accelerometer glued onto the engine block. Firstly, in order to develop a procedure for network training and for the selection of network parameters, the neural network is trained using a single data set, i.e. measurements taken at one operating point of the engine. In order to achieve computational savings and better network performance, most of the analysis was conducted using signals clipped to the interval of $\pm 30^{\circ} \mathrm{CA}$ around TDC. There, all relevant phenomena take place, and the correlation between signals is the highest. The results show that the choice of the spread parameter significantly affects the performance with the model. Hence, as the procedure involves a training of the network which turns out to be very quick, it is recommended to perform several runs, for example, for a fixed number of neurons while changing the basis spread, in order to establish the optimal combination with the number of neurons and the value of the spread parameter.

In a second phase, development and test of the neural network is conducted basing on data collected for a range of operating conditions, including different fuels and combustion modes. The network is then coded in a macro in a commercial indicating system, and successfully tested for on-line use. Results are promising in terms of performance and accuracy, both for the predicted pressure signals and for the pressure-derived engine parameters, which can be used in a closed-loop engine control system. We can conclude that robustness is verified with respect to changes in the engine operating conditions as well as with respect to changes in the nature of the fuel and of the combustion modes. The results indicate that the prediction model based on RBF neural network can be incorporated in the design of fuelindependent real-time control of Diesel engines.

\section{Acknowledgements}

The authors wish to thank Carlo Rossi and Bruno Sgammato for their precious help with the experimental apparatus. K. Bizon would like to acknowledge Università degli Studi del Sannio, where she was affiliated during the development of the present work.

\section{References}

[1] J.B. Heywood, Internal Combustion Engine Fundamentals, Mc Graw-Hill, New York, 1988

[2] N. Docquier, S. Candel, Combustion control and sensors: a review, Progress in Energy and Combustion Science 28 (2002) $107-150$. http://dx.doi.org/10.1016/S0360-1285(01)00009-0.

[3] O. Chiavola, G. Chiatti, L. Arnone, S. Manelli, Combustion characterization in diesel engine via block vibration analysis, SAE paper No. 2010-01-0168 (2010).

[4] F. Taglialatela Scafati, M. Lavorgna, E. Mancaruso, Use of vibration signal for diagnosis and control of a four-cylinder diesel engine, SAE paper No. 2011-24-0169 (2011).

[5] F. Taglialatela Scafati, N. Cesario, M. Lavorgna, E. Mancaruso, B.M. Vaglieco, Diagnosis and control of advanced diesel combustions using engine vibration signal, SAE paper No. 2011-01-1414 (2011).

[6] S. Saraswati, S. Chand, Reconstruction of cylinder pressure for SI engine using recurrent neural networks, Neural Computing and Applications 19 (2010) 935-944. http://dx.doi.org/10.1007/s00521-010-0420-6.

[7] F. Taglialatela Scafati, M. Lavorgna, E. Mancaruso, B.M. Vaglieco, Determination of combustion parameters using engine crankshaft speed, Mechanical Systems and Signal Processing 38 (2013) 628-633. http://dx.doi.org/10.1016/j.ymssp.2012.12.009.

[8] R. Johnsson, Cylinder pressure reconstruction based on complex radial basis function networks from vibration and speed signal, Mechanical Systems and Signal Processing 20 (2006) 1923-1940. http://dx.doi.org/10.1016/j.ymssp.2005.09.003.

[9] S. Leonhardt, C. Ludwig, R. Schwarz, Real-time supervision for diesel engine injection, Control Engineering Practice 3 (1995) $1003-1010$. http://dx.doi.org/10.1016/0967-0661(95)00084-8.

[10] I. Arsie, C. Pianese, M. Sorrentino, A procedure to enhance identification of recurrent neural networks for simulating air-fuel ratio dynamics in SI engines, Engineering Applications of Artificial Intelligence 19 (2006) 65-77. http://dx.doi.org/10.1016/j.engappai.2005.06.003.

[11] J. Wang, Y. Zhang, Q. Xiong, X. Ding, NOx Prediction by cylinder pressure based on RBF neural network in diesel engine, International Conference on Measuring Technology and Mechatronics Automation (2010) 792-795. http://dx.doi.org/10.1109/ICMTMA.2010.621.

[12] M. Yoon, K. Lee, M. Sunwoo, A method for combustion phasing control using cylinder pressure measurement in a CRDI diesel engine, Mechatronics 17 (2007) 469-479. http://dx.doi.org/10.1016/j.mechatronics.2007.06.001.

[13] Y GAO, R.B. Randall, Reconstruction of diesel engine cylinder pressure using a time domain smoothing technique, Mechanical Systems and Signal Processing 13 (1999) 709-722. http://dx.doi.org/10.1006/mssp.1999.1229.

[14] D. Moro, N. Cavina, F. Ponti, In-cylinder pressure reconstruction based on instantaneous engine speed signal, Journal of Engineering for Gas Turbines and Power 124 (2002) 220-225. http://dx.doi.org/10.1115/1.1391430.

[15] J. Antoni, J.Daniere, F. Gulle, Effective vibration analysis of IC engines using cyclostationarity. Part II new results on the reconstruction of the cylinder pressures, Journal of Sound Vibration 257 (2002) 839-856. http://dx.doi.org/10.1006/jsvi.2002.5063.

[16] D.S. Broomhead, D. Lowe, Multivariable functional interpolation and adaptive network, Complex Systems, 2 (1988) $321-355$.

[17] C. Harpham, C.W. Dawson, The effect of different basis functions on a radial basis function network for time series prediction: A comparative study, Neurocomputing 69 (2006) 2161-2170. http://dx.doi.org/10.1016/j.neucom.2005.07.010.

[18] S. Garg, K. Patra, S.K., Pal, D. Chakraborty, Effect of different basis functions on a radial basis function network in prediction of drill flank wear from motor current signals, Soft Computing, 12 (2008) 777-787. http://dx.doi.org/10.1007/s00500-007-0236-1.

[19] F.-L. Luo, R. Unbehauen, Applied neural network for signal processing, Cambridge University Press, Cambridge, 1998.

[20] I. Arsie, F. Marotta, C. Pianese, G. Rizzo, Information based selection of neural networks training data for S.I. engine mapping, SAE paper 2001-01-0561 (2001). 